For personal use only. Not to be reproduced without permission of the publisher (editorial@gabi-journal.net).

\section{Legislative efforts to limit prescrip- tion information sharing between patients and healthcare providers represent a serious threat to the health and safety of the American nation}

\author{
Janet SWyatt, RN, PhD, FAANP
}

Legislation in support of pharmacist notification to patients and providers of substitution of an interchangeable biologic for the originator biologic was recently vetoed in California. Automatic substitution of approved biosimilars without notifying the patient and physician/healthcare provider circumvents important pharmacovigilance.

Keywords: Biologicals, patients, REMS

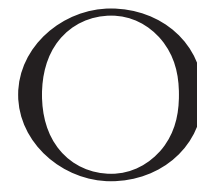
n 13 October 2013, Governor Jerry Brown ignored the recommendations of California legislators and caved into the pressure from outside groups to veto a bill that supported and strengthened patient-provider communication. At a time when national efforts are underway to increase public information sharing and transparency in the most complex corners of our government and society, California and many other state governments have now moved in the opposite direction with the promulgation of regulations which will allow pharmacists to exchange a prescribed biological product for a biosimilar product, without notifying the patient or the prescribing provider.

For those of us taking biological products for rheumatoid arthritis (RA) and other autoimmune diseases, this is appalling and scary. For almost 20 years the discovery and development of complex biological products has been a healthcare triumph for millions with RA and a business boon for pharmaceutical manufacturers who gain more than US $\$ 140$ billion in sales of biologicals annually. Investigation of the processes needed to manufacture biologicals helps to understand these costs. Biologicals products (medications) are a direct result of our growing knowledge of
DNA cloning. These complex treatments are genetically engineered proteins, made from animal, or bacteria cells. Biologicals require sophisticated production and purification processes and delicate handling. Today, there are as many as 10 separate biological products, delivered via self-injection or vein infusion that have helped improve the quality of life for people with RA. As patents on the highly expensive original biological therapies are due to expire in 2014, support is growing for the development of biosimilars, products that will be similar to and potentially interchangeable for original biologicals. While it is hoped that biosimilars might eventually reduce patient costs for biological therapy and increase access to safe biological treatments, it is important to recognize that any new biosimilar product will in no way be the same as the original biological. With the advent of genetic engineering that now transforms foreign cellular proteins into break-through treatments, we cannot apply the same assumptions and rules that govern the production and labelling of 'generic' medications. Even though the patent for a biosimilar may expire, biological drug manufacturers will not be required to turn over their original cell line or share information about their sophisticated manufacturing process. Essentially biosimilar manufacturers will not have access to an original biological's genetic engineering process or molecular clone and will not be able to access the original biological's active drug substance, production, fermentation, purification or delivery process. While the goal of interchangeable biosimilar therapy is to provide the same clinical benefit with no increased risks to patients, all new biosimilars will essentially be new products. Today, the US Food and Drug Administration (FDA) requires most if not all biologicals prescriptions to be accompanied by a detailed Risk Evaluation and Mitigation Strategy (REMS) which outlines specific monitoring and patient education that must be completed to support informed patient consent within the prescribing treatment process. Through REMS programmes, patients and providers engage in the valued concept of pharmacovigilance - monitoring for the benefits as well as the multitude of potential severe adverse reactions that accompany biological therapies. Indeed the importance of shared patient/provider pharmacovigilance was recently reinforced in the February 2013 FDA's Inspector General report which cited the need for strengthening patient and provider communication to improve monitoring of complex treatments. Thus, allowing substitution without notification will now severely undermine and complicate risk management strategies since patients and their physicians will not know which product(s) were used if/when adverse effects occur or which was responsible if many are used.

The decision to embark on biological therapy has always involved comprehensive communication between patients and providers. This has been particularly true for those of us with other illnesses and health conditions who are also taking other medications or treatments. But we cannot fool ourselves into thinking that biosimilar products are essentially generic versions of original biologicals. So if indeed new biosimilars are different cells, genetically engineered differently, manufactured and purified and produced differently, with potentially different side and adverse effects ... why eliminate the need for crucial patient and provider communication and health education?

A law that allows a pharmacist with no direct knowledge of your health conditions to change a biological for an interchangeable biosimilar without notifying

Author: Janet S Wyatt, RN, PhD, FAANP, 35460 Sassafras Drive, Round Hill, VA 20141, USA

Submitted: 21 October 2013; Revised: 22 October 2013; Accepted: 25 October 2013; Published online first: 7 November 2013 
the patient or the provider abandons the critical premise for pharmacovigilance defined by FDA - frequent and in-depth patient-provider communication.

With less than 20 years of accumulated patient response data, we are still learning about the benefits as well as the serious health risks of current biological therapies. As a patient with RA who has recently experienced an adverse reaction to a biological it is my hope that new biosimilars might bring new benefits. We do not have a cure for RA and we need all of the help we can get to manage the bone and joint erosion that marches on, despite the disease modifying effect of biologicals. But we cannot accept any regulation that circumvents the therapeutic relationship between patient and provider and abandons the important principles that guide safe, quality health care ... shared patient and provider communication.
Competing interests: None. Dr Janet S Wyatt is an independent consultant in health care, health policy and health advocacy. Dr Wyatt serves as a volunteer on the national Board of Directors of the Arthritis Foundation in the US.

Provenance and peer review: Not commissioned; internally peer reviewed.

DOI: 10.5639/gabij.2013.0204.045

Copyright (c) 2013 Pro Pharma Communications International 